\title{
Acetic acid disinfection as a potential adjunctive therapy for non-severe COVID-19
}

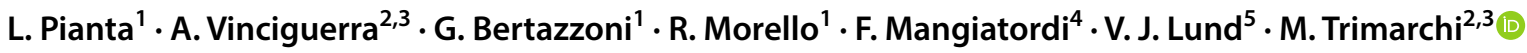

Received: 25 April 2020 / Accepted: 18 May 2020 / Published online: 24 May 2020

(c) Springer-Verlag GmbH Germany, part of Springer Nature 2020

\begin{abstract}
Purpose SARS-CoV-2 is a new pandemic influenza caused by a coronavirus which main route of transmission is through exhaled droplets that primarily infect the nose and the nasopharynx. The aim of this paper is to evaluate the effect of acetic acid, the active component of vinegar, as a potential disinfectant agent for upper airways.

Methods Twenty-nine patients were enrolled and divided into two groups: group 1 (14 patients) was composed of patients treated with off-label hydroxychloroquine and lopinavir/ritonavir, whereas group 2 (15 patients) was composed of patients treated with hydroxychloroquine only, combined with the inhalation of acetic acid disinfectant at a $0.34 \%$ concentration. A questionnaire-based evaluation of symptoms was performed after 15 days in both groups.

Results It appears that the number of patients treated with acetic acid (group 2) that experienced improvement in individual symptoms was double that of the other group of patients (group 1), although numbers are too small for robust statistical analysis.

Conclusions Considering its potential benefits and high availability, acetic acid disinfection appears to be a promising adjunctive therapy in cases of non-severe COVID-19 and deserves further investigation.
\end{abstract}

Keywords COVID-19 $\cdot$ Acid acetic $\cdot$ Disinfection $\cdot$ Coronavirus

\section{Introduction}

The main route of transmission of the new coronavirus called COVID-19 (SARS-CoV-2) is through mucosal contact with infected exhaled droplets [1]. Considering the higher viral load detected in the nose, compared with the lower respiratory tract [2], this may be one of the first anatomical structures exposed to viral contagion as evidenced

M. Trimarchi

trimarchi.matteo@hsr.it

1 Department of Otorhinolaryngology, ASST Cremona, Cremona, Italy

2 Division of Head and Neck Department, Otorhinolaryngology Unit, San Raffaele Hospital, IRCCS San Raffaele Scientific Institute, Via Olgettina, 68, 20100 Milan, Italy

3 School of Medicine, Vita-Salute San Raffaele University, Milano, Italy

4 Department of Emergency, ASST Cremona, Cremona, Italy

5 Royal National Throat, Nose and Ear Hospital, UCLH, London, UK by the fact that acute anosmia is a relatively common presenting symptom [3, 4]. With the rapid spread of COVID-19, global health-care systems have faced the challenge of treating an overwhelming number of patients for which there is no widespread immunity. As a result, many off-label therapies have been tried but so far with uncertain results. In addition, the mild symptoms in the majority of cases and the saturation of hospital beds may compel doctors to treat the majority of infected people at home. In this situation, experimental treatments are not readily available to those at home or be available in low-resource setting [5].

Acetic acid, the active component of vinegar, is a commonly available disinfecting agent. Inhalation of a waterbased acetic acid solution to treat the symptoms of the common cold is a common folk remedy in Italy. Indeed, the anti-bacterial and anti-viral activities of acetic acid are documented in the literature [6-9]. Acetic acid causes inactivation and dis-aggregation of haemagglutinin glycoproteins (found on the surface of influenza viruses) by generating a low $\mathrm{pH}$-dependent conformational change of those glycoproteins and it destroys the viral envelope and inhibits viral transmission [6]. Therefore, considering these antiviral 
properties and that the upper airways are the main site of SARS-CoV-2 entry and replication, we have explored the use of this historical therapy in early-stage cases of COVID19 by evaluating patients who were administered a disinfecting formulation of acetic acid as an intranasal aerosol.

\section{Materials and methods}

In this prospective study, we included patients attending the Emergency Department of Cremona Hospital with positive real-time polymerase chain reaction (RT-PCR) for SARSCoV-2 performed on nasopharyngeal swabs.

The study was conducted according to the ethical standards established in the 1964 Declaration of Helsinki (revised in 2013) and was approved by the local ethics committee (Comitato etico Val Padana, Protocol No. 63-2020-OSS_ALTRO-CR45).

Records of patients positive for COVID-19 infection with mild symptoms who were discharged home after accessing the Emergency Room over March 2020 were evaluated and included in the study. Exclusion criteria were asthma (due to the possible bronchoconstriction from the acetic fumes) and intolerance to sulphites.

Patients treated with off-label hydroxychloroquine and lopinavir/ritonavir, the standard treatment prescribed at that time, were included in group 1, whereas group 2 was composed of patients treated with hydroxychloroquine only, combined with acetic acid nasal disinfectant at a $0.34 \%$ concentration.

While group 2 was created prospectively, group 1 was composed of patients that were recorded in the same period but retrospectively included in the study.

The solution was prepared by patients at home, using 3.5 dessert spoons (approximately $35 \mathrm{ml}$ in total) of vinegar (with a 6\% concentration of acetic acid) in $500 \mathrm{ml}$ of boiling water. Patients were instructed to exclusively inhale the fumes of the solution through the nose using a commercially available device (Fig. 1) for 10 min twice a day. Inhalations could also be performed using a bowl with a towel over the head, with the only precaution of covering eyes to avoid possible local irritation.

Patients' symptoms at presentation, clinical course, symptoms after 15 days and the presence of SARS-CoV-2 on nasopharyngeal swabs taken after 15 days were recorded. Symptoms were evaluated through a questionnaire based on general questions on comorbidities, general and ENT symptoms associated with COVID-19 infection. The questionnaire only offered a binary answer (yes/no) to the following questions: Do you have/experienced any improvement/ resolution of Cough? Fever? Shortness of breath? Vomiting/ Diarrhoea? Fatigue during the day? Headache? Nasal sneezing? Blockage/Congestion of the nose? Loss of smell/taste?
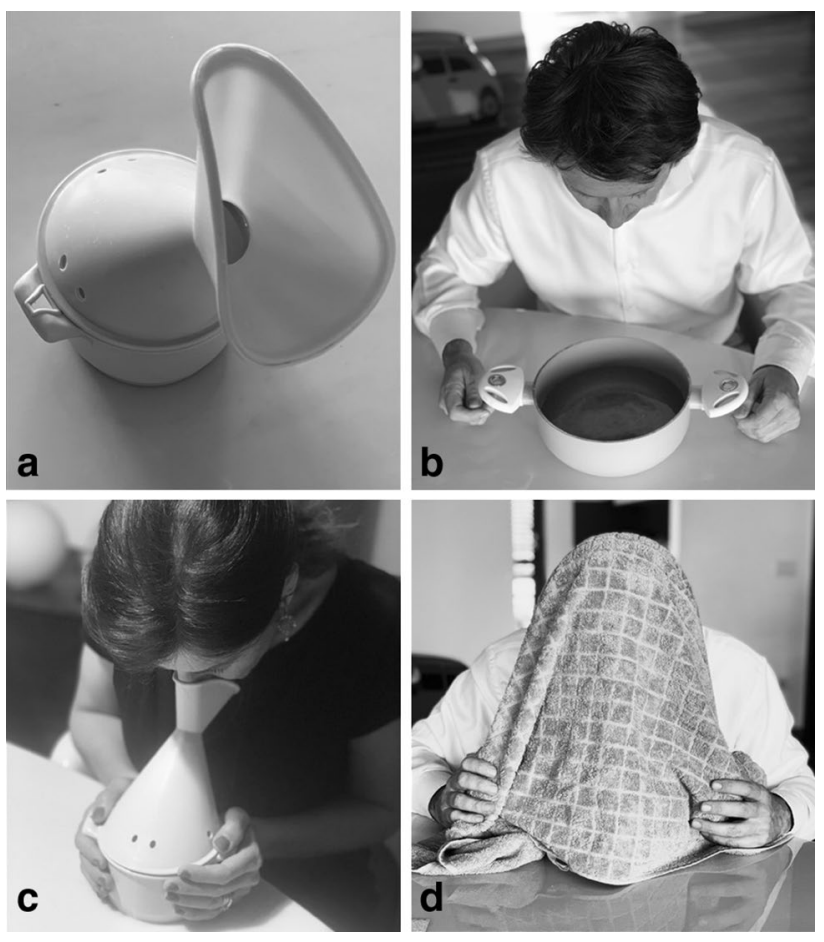

Fig. 1 An inhalation plastic device is shown (a). The mask is applied to the face to inhale the disinfectant solution (c). The acetic acid solution can be poured in a bowl (b) and fumes are inhaled placing the face above the bowl; the head is covered by a cloth to reduce fume dispersion (d)

\section{Results}

Twenty-nine patients were enrolled. There were 14 patients in group 1 (standard treatment) ( 7 females and 7 males, aged 33-73 years), and 15 patients in group 2 (acetic acid treatment) (11 males and 4 females, aged $49-80$ years). Treatment compliance was $100 \%$ in both groups; no adverse events related to acetic acid inhalation were reported.

In group 1, all patients presented cough and fever, whereas in group 2, seven patients reported mild cold symptoms and eight patients cough and fever. All patients were followed for 15 days. In group 1 , one patient was hospitalized because of increasing severity of respiratory symptoms, while 13 patients reported improvement of symptoms after 15 days. Seven patients out of 13 had a negative RT-PCR for COVID-19 at 15 days.

In group 2, after 15 days, all patients reported symptom improvement, $12 / 15$ patients tested negative for SARSCoV-2, whereas $3 / 5$ patients were still positive. Twice the number of patients experienced improvement in individual symptoms in group 2 than group 1 though numbers are too small for statistical analysis. (Table 1).

In all cases, the acetic acid inhalation was well tolerated as evidenced by compliance, although this was self-reported. 
Table 1 Patient's questionnaire responses before (pre) and after (post) the therapy

\begin{tabular}{lllll}
\hline & $\begin{array}{l}\text { Group 1 pre } \\
N=14\end{array}$ & Group 1 post & $\begin{array}{l}\text { Group 2 pre } \\
N=15\end{array}$ & Group 2 post \\
\hline Cough & 11 & 1 & 12 & 0 \\
Fever & 13 & 2 & 8 & 0 \\
Dyspnoea & 3 & 2 & 2 & 0 \\
Vomit/diarrhoea & 1 & 0 & 1 & 0 \\
Fatigue during the day & 6 & 5 & 6 & 3 \\
Headache & 3 & 2 & 4 & 0 \\
Blockage/congestion of the nose & 7 & 3 & 7 & 2 \\
Anosmia & 4 & 2 & 5 & 2 \\
Dysgeusia & 2 & 2 & 2 & 1 \\
\hline
\end{tabular}

Group 1 control group, Group 2 vinegar group

\section{Discussion}

The results of the study suggest a possible effect of acetic acid on the upper respiratory airway, which may lead to a faster clinical resolution of COVID-19. Our study is one of the first attempts to identify a topical therapy that could influence the natural history of COVID-19. The efficacy of acetic acid could be attributed to the exposure of respiratory mucosa, a known hotspot of viral replication, to a highly effective disinfectant. Acetic acid disinfection could not only improve the clinical course of COVID-19, but also reduce viral load in the airway and in the droplets exhaled by infected individuals. Thus, a possible beneficial effect of this topical treatment in reducing the spread of the disease can also be hypothesized.

The decision to avoid anti-viral therapies in group 2 was based on the increasing concern about the real benefits of that treatment and in the updated Italian guidelines (March 2020) [10], ritonavir/lopinavir is now not considered to be effective. Therefore, taking into account the ineffectiveness of ritonavir/lopinavir, the pharmacological treatment regimens of the two groups can be considered equivalent.

This study have (has) some limitations: first, it would have been important to have a visual analogue scale to evaluate symptoms referred by the patients; second, some factors like age and gender differences between studied group could be considered a bias in the outcome of the study; third, due to the emergent situation, no anosmia testing was performed in the Emergency Department so that it is only based on self-reported symptom.

In conclusion, considering its potential benefits, high availability and absence of reported side effects, acetic acid disinfection appears to be a promising, cheap, easily administered and well-tolerated adjunctive therapy in cases of nonsevere COVID-19.

This is a small study performed at the height of the COVID crisis in a rapidly changing clinical environment. Additional studies on larger samples with a control inhalation arm are needed to provide high-quality evidence to further support the employment of this ancient remedy.

Author contributions LP and MT made substantial contribution to the concept and design of the work; GB and RM contributed to the data acquisition and its analysis; AV took his part in the interpretation of data and in drafting the article; VJL participated in revising the article critically and gave the final approval of the version to be submitted.

Funding This research received no specific grant from any funding agency in the public, commercial, or not-for-profit sectors.

\section{Compliance with ethical standards}

Conflict of interest None of the authors has any conflict of interest.

Ethical approval Approval was obtained from the ethics committee of Val Padana (No.63-2020-OSS_ALTRO-CR45). The procedures used in this study adhere to the tenets of the Declaration of Helsinki.

Informed consent Informed consent was obtained from each patient for treatment and use of de-identified clinical data for study purposes.

\section{References}

1. Han Y, Yang H (2020) The transmission and diagnosis of 2019 novel coronavirus infection disease (COVID-19): a Chinese perspective. J Med Virol. https://doi.org/10.1002/jmv.25749

2. Zou L, Ruan F, Huang M et al (2020) SARS-CoV-2 viral load in upper respiratory specimens of infected patients. N Engl J Med 382:1177-1179. https://doi.org/10.1056/NEJMc2001737

3. Eliezer M, Hautefort C, Hamel AL et al (2020) Sudden and complete olfactory loss function as a possible symptom of COVID19. JAMA Otolaryngol Head Neck Surg. https://doi.org/10.1001/ jamaoto.2020.0832

4. Hopkins C, Surda P, Kumar N (2020) Presentation of new onset anosmia during the COVID-19 pandemic. Rhinology. https://doi. org/10.4193/Rhin20.116

5. Greatorex JS, Page RF, Curran MD et al (2010) Effectiveness of common household cleaning agents in reducing the viability of human influenza A/H1N1. PLoS ONE 5:e8987. https://doi. org/10.1371/journal.pone.0008987 
6. Alphin RL, Johnson KJ, Ladman BS, Benson ER (2009) Inactivation of avian influenza virus using four common chemicals and one detergent. Poult Sci 88:1181-1185. https://doi.org/10.3382/ ps.2008-00527

7. Fraise AP, Wilkinson MA, Bradley CR, Oppenheim B, Moiemen N (2013) The antibacterial activity and stability of acetic acid. J Hosp Infect 84:329-331. https://doi.org/10.1016/j. jhin.2013.05.001

8. Halstead FD, Rauf M, Moiemen NS et al (2015) The antibacterial activity of acetic acid against biofilm-producing pathogens of relevance to burns patients. PLoS ONE 10:e0136190. https://doi. org/10.1371/journal.pone.0136190

9. Rabenau HF, Cinatl J, Morgenstern B, Bauer G, Preiser W, Doerr HW (2005) Stability and inactivation of SARS coronavirus.
Med Microbiol Immunol 194:1-6. https://doi.org/10.1007/s0043 0-004-0219-0

10. Società Italiana di Malattie Infettive e Tropicali (SIMIT) (2020) Vademecum per la cure delle persone con malattia da COVID-19. Italian Guidelines, 2 ed. March 2020

Publisher's Note Springer Nature remains neutral with regard to jurisdictional claims in published maps and institutional affiliations. 